\title{
The Effective Optimization Methods of Port Activity on the Basis of Algorithmic Model
}

\author{
A. Nyrkov, A. Shnurenko, S. Sokolov, S. Chernyi, V. Korotkov \\ Departement Engineering, Admiral Makarov State University of Maritime and Inland Shipping, Russia
}

\begin{tabular}{l} 
Article Info \\
\hline Article history: \\
Received May 3, 2017 \\
Revised Aug 5, 2017 \\
Accepted Aug 25, 2017 \\
\hline
\end{tabular}

Keyword:

Waterways

Cargo

Vessel

Optimization

\begin{abstract}
The article considers the inland waterways (IW) within the international transport corridors as multiphase systems of mass service (SMS) and shows the distinction in calculation of the important indicator of system efficiency the average total waiting time of vessel service in the serving subsystems, the application of genetic algorithms to increase the efficiency of reloading processes.
\end{abstract}

Copyright @ 2017Institute of Advanced Engineering and Science. All rights reserved.

\section{Corresponding Author:}

Sergei Chernyi,

Admiral Makarov State University of Maritime and Inland Shipping

Sankt-Peterburg, Russia

Email: sergiiblack@gmail.com

\section{INTRODUCTION}

The market relations impose to river transport the strict requirements on acceleration of cargo and passengers delivery at the smallest costs of transportation, on decrease of the transport component in prime cost of production, on improvement of quality and reliability of transportations. The current trends of transport services development compel the various types of transport to pass from direct fierce competition among themselves to more mutually beneficial cooperation within intermodal transportations. Development of such transportations in Russia is closely connected with situation on the all-European market of goods and transport services.

A significant role in the integration processes belongs to the creation of conditions for free rendering of services on international transportations river-sea. The river transport provides the realization of transit potential of Russia within the international transport corridors. The integration world processes directly affect the interests of the Russian Federation which according to the Partnership and Cooperation Agreement with the EU assumed certain obligations for opening of inland waterways for the international navigation. The transport corridors are elements of the international logistic structure. They are intended for achievement of bigger efficiency of transport process that is provided by application of the latest developments in technic, technology of the organization of transport and reloading process, and also by creating of favorable conditions for the transportations implementation.

The transport corridors are the powerful trunklines corresponding to the requirements of the international standards with uniform technological organizational legal norms and conditions. They allow to achieve considerable decrease in terms and cost of transportations, increase of their quality and reliability. The active integration into the system of the international transport corridors creates the additional opportunities for development of the Russian transport system and further improvement of its industrial, information and technological infrastructure. 
The uniform technology of cargoes transportation in the transport corridors assumes a continuity of the transport process with minimization (elimination) of faulty situations, first of all, in the reloading points (distribution centers) and, including, in river ports. Such system demands the optimization of distribution centers, definition of the optimum transportations plan, detailed calculations of types and number of vehicles, cargo handling equipment and other resources, as well as coordination of different work technologies of each type of transport, work coordination of the trunkline transport and cargo owners in the transfer points. All these issues are solved by using the methods of "physical" distribution of cargoes, logistics and economicmathematical simulation. However, besides the traditional economic-mathematical methods for efficiency calculation of similar systems it is possible to use the mathematical apparatus of the mass service theory and also the algorithms of discrete mathematics [1-3].

\section{INLAND WATERWAYS AS MULTIPHASE MASS SERVICE SYSTEMS (MSS)}

We will consider the river system including initial, final and the intermediate transit points connected by river arteries, channels and locks. From the point of view of the mass service theory, the similar system (or less difficult) can be considered as set of the mass service systems (MSS) with expectation which are consistently connected with each other so that the flow of the served requests (vessels) coming out from one system is the flow coming in the following system. Such systems connection is multiphase MSS with expectation. Each component of system is called as a phase. The incoming flow of requests for multiphase MSS is the stream coming in the first phase; the outcoming flow is the stream coming out from the last phase (Figure 1).

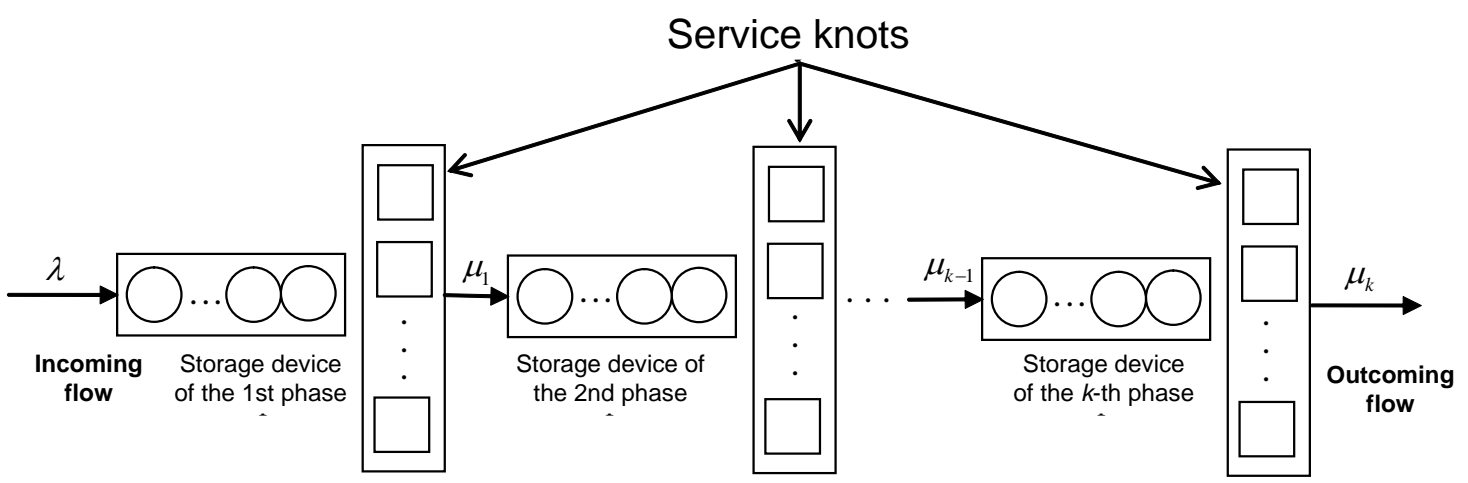

Figure 1. The scheme of multiphase MSS with expectation

The distribution of service channels set to some groups (service knots) is characteristic for systems of multiphase service. The transit points (river ports) and locks can be considered as knots on the inland waterways [4]. Assume that the incoming flow of vessels is close to the simplest, and the service time on each phase is distributed according to a random law with mean value $\bar{t}_{o b s}$ and standard deviation $\sigma_{t}$. In this case the flow intensity of the served vessels is $\mu=1 / \bar{t}_{\text {obs }}$. Assume that there are $\mathrm{k}$ phases of service in a system. The simplest flow of vessels with intensity $\lambda$ comes to the system. After requests processing on the first phase (for example, loading in the initial transit point) the service knot (river or maritime port) forms the flow of the served requests with intensity $\mu_{1}=1 / \bar{t}_{o b s, 1}$. This flow is incoming for the second phase of system (for example, passing of the lock No. 1). After the second knot of service channels the flow of the served requests with intensity $\mu_{2}=1 / \bar{t}_{o b s, 2}$ is formed, that is incoming for the third phase, etc. After all requests passed the processing of k- service knot, the flow of the served requests leaves a system with intensity $\mu_{k}=1 / \bar{t}_{\text {obs }, k}[5]$.

All flows in the system are the simplest, and therefore without consequences. Therefore the whole system can be considered as consisting of $\mathrm{k}$ subsystems. The own intensity of request flow will correspond each subsystem: $\alpha_{1}=\lambda / \mu_{1}, \alpha_{2}=\mu_{1} / \mu_{2}, \ldots, \alpha_{k}=\mu_{k-1} / \mu_{k}$. Steady state of the system operation will be possible under conditions when $\alpha_{i}<1$, where $i=1,2, \ldots, k$. 
The average waiting time of request service $\left(\bar{t}_{\text {ож⿻ }}\right)$ is one of the most important efficiency indicators of system operation. According to Polyachek-Hinchin's formula $\bar{t}_{\text {ож }}=\frac{\alpha^{2}\left(1+v^{2}\right)}{2 \lambda(1-\alpha)}$, where $\alpha-$ intensity of requests flow, $\lambda$-intensity of incoming requests flow, $v=\sigma_{t} / \bar{t}_{\text {obs }}-$ coefficient of service time variation.

If we consider each service phase as the separate system independent of others, the general waiting time is equal to the sum of durations of expectation on each transit point: $\bar{t}_{\hat{\imath} j}=\sum_{i=1}^{k} \bar{t}_{\hat{\imath} j, i}$.

If we consider the transport water corridor as uniform multiphase system, at calculation it is necessary to consider phase coefficient $f=\frac{k_{f}+1}{2 k_{f}}$, where $k_{f}$ - number of phases in the system [6].

When the phase quantity increases, the phase coefficient decreases asymptotically approaching 0.5 . The physical meaning of this statement is that the general waiting time of service at several phases of service at the expense of f-coefficient will be less, than the sum of expectation duration separately by each type of service, i.e. $\bar{t}_{\hat{\imath} j}^{*}=f \cdot \bar{t}_{\hat{\imath} j}=f \cdot \sum_{i=1}^{k} \bar{t}_{\hat{\imath} j, i}<\bar{t}_{\hat{\imath} j}$.

It is explained by that in process of transition from one phase to other the vessels flow becomes more ordered. The process of cargos acceptance on the service gradually loses the stochastic nature and becomes systematic. Thus, considering the river transport system as multiphase MSS, it is possible to receive more natural efficiency indicators of its functioning. Such approach allows to plan more favourably the distribution of powers on the nodal points of the transport corridor [7-9].

\section{THE ALGORITHMS OF EFFICIENCY IMPROVEMENT OF RELOADING PROCESSES}

Deficiency of warehouse spaces is observed almost in all ports therefore their effective use promises considerable benefits for transshipment complexes. This problem is connected with the rational distribution on warehouse spaces of a port, and also the reduction of periods of cargoes storage in a port. At the same time the problems solution of cargoes placement is applicable not only for warehouse operations. The similar procedures can be applied, in particular, for the tasks of rational completing of freights at a cargo-planning. In general, the task of optimal placement of cargoes can be described in the following way:

- there are $\mathbf{N}=\left\{t_{1}, t_{2}, \ldots, t_{n}\right\}$ cargoes various or identical in a form, dimensions and physical characteristics (mass, density, and others);

- $\quad$ there are $\mathbf{M}=\left\{p_{1}, p_{2}, \ldots, p_{m}\right\}$ volumes provided for the cargoes placing;

- $\quad$ a set of parameters and restrictions imposed on placement process is given;

- two types of tasks are possible:

1. Maximally possible quantity of cargoes from $\mathbf{N}$ is located in $\mathbf{M}$;

2. To locate the whole cargo from $\mathbf{N}$ in $\mathbf{M}$ occupying the space as less as possible.

Both tasks belong to NP-full tasks for which there is no uniform solution algorithm. At the same time the special types of these tasks are solved by various algorithms suitable only for specific tasks. In most cases it is heuristic algorithms. We will consider the special type of the placement task.

The initial data:

1. There are $K$ vessel bunkers $-\mathbf{D}=\left\{d_{1}, d_{2}, \ldots, d_{K}\right\}$ (rectangular parallelepipeds) with the linear sizes: $H_{1}, H_{2}, \ldots, H_{K}-$ their heights, $A_{1}, A_{2}, \ldots, A_{K}-$ their lengths, $B_{1}, B_{2}, \ldots, B_{K}-$ their widths.

2. The containers types (the special case is three types): $h_{1}, h_{2}, h_{3}$ - heights of the first, second, third type respectively, $a_{1}, a_{2}, a_{3}$ - the containers lengths, $b_{1}, b_{2}, b_{3}$ - the containers widths.

3. $\mathbf{N}=\bigcup_{i=1 . . n} n_{i}-$ plurality of all containers assumed for loading. $n_{1}=\left\{l_{11}, l_{12}, \ldots, l_{1 x}\right\}$, $n_{2}=\left\{l_{21}, l_{22}, \ldots, l_{2 y}\right\}, n_{3}=\left\{l_{31}, l_{32}, \ldots, l_{3 z}\right\},-$ pluralities consisting of containers each of three types. $U=x+y+z-$ the total number of containers. $\mathbf{C}_{\mathbf{i}}-$ the plurality reflecting the value of the containers 
of i-type assumed for loading.

4. $\mathbf{W}=\left\{m_{1}, m_{2}, \ldots, m_{U}\right\}-$ the mass of the containers assumed for loading. $\mathbf{M}=\sum_{i=1}^{U} m_{i}-$ the total mass of container.

5. $\mathbf{P}$ - most acceptable mass of cargo for this type of vessel.

6. $\mathbf{O}$ - value of possible deviation of the sums of cargoes masses located on the opposite sides of the vessel, in tons (in fact this value represents the maximum acceptable ballast mass for providing of the vessel stability).

7. $\mathbf{Q}=\left\{s_{1}, s_{2}, \ldots, s_{U}\right\}$ - the additional plurality (not obligatory, but desirable) containing the containers sequence for loading sorted in decreasing order of the cargoes importance in the container.

The rules of placement and requirement of completing:

1. It is possible to put at each other only containers of one type. The total mass of the containers for loading must not be more than the maximum acceptable cargo mass for this type of vessel, $\mathbf{V}=\left\{m_{1}, m_{2}, \ldots, m_{U}\right\}$ - the masses of the containers for loading as a result of the solution of the task.

2. The sides of the containers have to be parallel to the sides of the bunker.

3. The value of a possible mass difference of the cargoes located on the opposite sides of the vessel has to correspond to the recommended value according to the technical documentation on the vessel.

For the rational cargo placement in the selected space taking into account the stability we divide the containers plurality into four subsets: $\mathbf{F}_{\mathbf{1}}, \mathbf{F}_{\mathbf{2}}, \mathbf{F}_{\mathbf{3}}, \mathbf{F}_{\mathbf{4}}$. These pluralities are the containers for loading in bunkers of four parts of the vessel received when splitting the vessel in a longitudinal and cross way. If any bunker is located on the line of splitting, then, figuratively speaking we divide it into two or four (in case of the central bunker) areas and we consider it further as two (or four) separate bunkers, and then we unite them at the decision receiving. The mass difference between four new containers pluralities has to meet the requirements of efficiency in the field of vessel stability.

Further for each bunker we define its relation to the containers plurality $\mathbf{F}_{\mathbf{t}}$. We divide $\mathbf{F}_{\mathbf{t}}$ into plurality of piles $-\mathbf{E}_{\mathbf{t}}=\left\{S t_{1}, S t_{2}, . ., S t_{d}\right\}$ in $H_{i} / h_{j}$ containers in each, where $i$ - number of the current bunker, $H_{i}$ - height of $i$-bunker, $j$ - number of the current type of containers, $h_{j}$ - height of the current type of container. Pile is formed from containers of one type following in the $\mathbf{C}_{\mathbf{i}}$ list in decreasing order of worth. The worth of pile is determined by the sum of worth of its all containers.

For the local optimum search it is offered to use the genetic algorithm with the "improved left lower" decoder:

1. To create the initial population from $R$ individuals. Each individual $T_{b}(b=1 . R)$ is obtained by the random rearrangement of numbers of the containers piles from the $\mathbf{E}_{\mathbf{t}}$ list.

2. To make the interbreeding of all individuals with the partner, random for each individual, uniting two pluralities of the piles received by results of item 1 implementation: $\mathbf{Y}=T_{p} \bigcup T_{q}(p, q \in R)$.

Then, we divide the resulting plurality into two (from two parental individuals we have two descendants) and we save all descendants.

3. To calculate the value of adaptation for each new individual, including descendant, by the decoding procedure: $f(\mathbf{Y})=\operatorname{decoder}(\mathbf{Y})$.

4. To order on decrease the values of adaptations and to leave the best individuals $O$ (the parameter is set separately).

5. To apply the mutation operator to each individual with small probability $\mu$.

6. To repeat items 2-5 until the highest value of the adaptation function in population doesn't stop to change. To provide two more ways of the evolution completion: when $O$ generations was executed and cancelability.

After application of the genetic algorithm we will receive the scheme of the piles placement in a hold. At this stage it is possible to determine the worth of the hold, as the sum of worth of the container piles located in it. 
Further we apply the genetic algorithm until all holds won't be filled or all containers won't be shipped. The plurality $\mathbf{Q}=\left\{s_{1}, s_{2}, \ldots, s_{U}\right\}$ also demands adjustment at all stages.

As a result of the genetic algorithm application and considering the possibility of non-compliance with consecutive filling of bunkers, according to sequence in the bunkers array $\mathbf{D}=\left\{d_{1}, d_{2}, \ldots, d_{K}\right\}$, we will receive a tree where weight of tops reflects the worth of the vessel bunkers.

\section{CONCLUSION}

Passing on the tree in search of the most rational decision meeting the requirements of efficiency, applying such algorithms as "search with exclusions", "annealing imitation", "ant algorithm", we will receive a set of decisions. From the received decisions it is necessary to make a choice of the most rational solution, perhaps, with revision of the efficiency requirements.

\section{REFERENCES}

[1] M. Ju. Alehin, etc. "Application of the mass service theory for the solution of production tasks", LKI, 1989.

[2] I.K. Volkov, S.M. Zuev, G.M. Tsvetkova, "Random processes: The textbook for higher education institutions", Publishing house of Bauman MSTU, 448, 2000.

[3] N. Dzhejsuol "Queues with priorities: translated from English by I.S. Nefedov and V.S. Manusevich", Publishing house World, 280, 1973.

[4] N.Sh. Kremer, B.A. Putko, I.M. Trishin, M.N. Fridman; ed.prof. N.Sh. Kremer, "Operations research in economics: Manual for higher education institutions", JuNITI, 407, 2002.

[5] A.V. Kirichenko, "Transportation of export-import cargoes. Organization of logistic systems", Piter, 506, 2004.

[6] A.P. Nyrkov, A.S. Belousov, S.S. Sokolov, "Algorithmic support of optimization of multicast data transmission in networks with dynamic routing", Modern Applied Science, 10(5), 162-176, 2015.

[7] Z.V. Boriev, S.S. Sokolov, A.P. Nyrkov, "Review of modern biometric user authentication and their development prospects", IOP Conference Series: Materials Science and Engineering, 91(1). DOI: 10.1088/1757899X/91/1/012063.

[8] Chernyi S. Techniques for selecting topology and implementing the distributed control system network. IOP Conference Series: Materials Science and Engineering, 124, (2016) 012048.

[9] Chernyi S. Use of Information Intelligent Components for the Analysis of Complex Processes of Marine Energy Systems. Transport and Telecommunication Journal, 17(3), (2016) 202-211. DOI: 10.1515/ttj-2016-0018 